\title{
Solar Radiation Pressure Models for BeiDou-3 I2-S Satellite: Comparison and Augmentation
}

\author{
Chen Wang ${ }^{1}$, Jing Guo ${ }^{1,2}{ }^{(0)}$, Qile Zhao ${ }^{1,3, *}$ and Jingnan Liu ${ }^{1,3}$ \\ 1 GNSS Research Center, Wuhan University, 129 Luoyu Road, Wuhan 430079, China; \\ chen.wang@whu.edu.cn (C.W.); jingguo@whu.edu.cn (J.G.); jnliu@whu.edu.cn (J.L.) \\ 2 School of Engineering, Newcastle University, Newcastle upon Tyne NE1 7RU, UK \\ 3 Collaborative Innovation Center of Geospatial Technology, Wuhan University, 129 Luoyu Road, \\ Wuhan 430079, China \\ * Correspondence: zhaoql@whu.edu.cn; Tel.: +86-027-6877-7227
}

Received: 22 December 2017; Accepted: 15 January 2018; Published: 16 January 2018

\begin{abstract}
As one of the most essential modeling aspects for precise orbit determination, solar radiation pressure (SRP) is the largest non-gravitational force acting on a navigation satellite. This study focuses on SRP modeling of the BeiDou-3 experimental satellite I2-S (PRN C32), for which an obvious modeling deficiency that is related to SRP was formerly identified. The satellite laser ranging (SLR) validation demonstrated that the orbit of BeiDou-3 I2-S determined with empirical 5-parameter Extended CODE (Center for Orbit Determination in Europe) Orbit Model (ECOM1) has the sun elongation angle ( $\varepsilon$ angle) dependent systematic error, as well as a bias of approximately $-16.9 \mathrm{~cm}$. Similar performance has been identified for European Galileo and Japanese QZSS Michibiki satellite as well, and can be reduced with the extended ECOM model (ECOM2), or by using the a priori SRP model to augment ECOM1. In this study, the performances of the widely used SRP models for GNSS (Global Navigation Satellite System) satellites, i.e., ECOM1, ECOM2, and adjustable box-wing model have been compared and analyzed for BeiDou-3 I2-S satellite. In addition, the a priori SRP models are derived based on analytical cuboid box model and empirically spectra analysis, respectively. Use of the a priori model combined with ECOM1 was finally demonstrated to reduce the $\varepsilon$-angle-dependent systematic error, and thus improved the radial orbit accuracy by nearly 35 per cent when compared to the solution with standalone ECOM1, as revealed by the one way SLR residuals.
\end{abstract}

Keywords: BeiDou-3 I2-S; precise orbit determination; solar radiation pressure; box-wing

\section{Introduction}

Five years after the official announcement of providing the positioning, navigation and timing (PNT) service around Asia-Pacific region, Chinese BeiDou Navigation Satellite System (BeiDou) has stepped into a new era recently. On 6 November 2017, two new-generation BeiDou satellites (BeiDou-3) have been successfully launched to medium earth orbit (MEO) to provide the global PNT service. Once the space segment is successfully deployed, BeiDou will possess global service capability comprising totally 35 satellites distributed in geostationary orbit (GEO), inclined geosynchronous orbit (IGSO) and MEO around 2020. In order to validate the new features of BeiDou-3 satellites, including new signals, inter-satellite link technology, and onboard frequency standards, two IGSO and three MEO experimental satellites have been launched.

Up to now, the published in-orbit-validation (IOV) results have demonstrated the significant improvements of BeiDou-3 satellites w.r.t the regional BeiDou satellites (BeiDou-2). The inter-satellite link technology does not only facilitate high accuracy precise orbit determination (POD), but also enables autonomous navigation as well as improves the independency and stability of BeiDou system [1]. The quality of BeiDou-3 signals is comparable to that of GPS L1/L2/L5 and Galileo 
E1/E5a/E5b signals, and the elevation-dependent code biases, which have been previously identified to exist in the code observations of the BeiDou-2 satellites, seem to be not obvious for BeiDou- 3 satellites, as demonstrated in [2]. These improvements ease the precise data processing of BeiDou. The stability of onboard frequency standards has been improved by a factor of 10 , and can be compared to the latest type of rubidium atomic frequency standards employed onboard the GPS IIF satellites, as well as the passive hydrogen masers used onboard the Galileo satellites. A continuous yaw-steering (YS) attitude model is used to avoid the significant orbit accuracy degeneration when a satellite switches its attitude mode [3]. Thanks to the above improvements, the better POD performance has been achieved for BeiDou-3 experimental satellites, except for BeiDou-3 I2-S (PRN C32) satellite, for which an apparent linear sun elongation angle ( $\varepsilon$ angle) dependent systematic error has been observed in the satellite laser ranging (SLR) residuals.

Without any doubt, the systematic error is original form the deficiency of non-gravitational force models, particularly in the solar radiation pressure (SRP) model. The similar error was also observed for European Galileo and Japanese QZSS Michibiki satellite's orbits determined with the 5-parameter extended CODE orbit model (called ECOM1 in this study) [4,5]. To reduce the deficiency of the ECOM1 for the Galileo satellites, an a priori SRP model based on a generic box-wing model (hereafter called cuboid model) in YS mode to augment the ECOM1 was established [6]. With this a priori model, the peak magnitude of radial orbit errors was reduced from presently $20 \mathrm{~cm}$ down to $5 \mathrm{~cm}$ for Galileo IOV satellites outside eclipse phases, and similar performance has also been achieved for Galileo Full-Operational-Capability (FOC) satellites [7]. With approximate body dimensions, optical properties as well as mass, the QZSS Michibiki's a priori cuboid model has been obtained, which improves the orbit to a better than $10 \mathrm{~cm}$ RMS consistency with SLR measurements [8]. The reasons for that type of model are that Galileo and QZSS Michibiki satellites have a markedly elongated shape instead of a cuboid one, as well as a large area-to-mass ratio. Alternatively, the revised version of ECOM (ECOM2) also has the potential to reduce such kind of error, i.e., for Galileo and QZSS Michibiki satellites [9], although the primer aim of the model is to reduce the draconitic errors in GNSS geodetic products [10]. Besides, the adjustable box-wing model (ABW) can also reduce this error, because the model is derived from the physical theory, and can fit real tracking data well, as shown for QZSS Michibiki satellite [11].

In this study, the performances of the above mentioned ECOM1, ECOM2, and ABW models for BeiDou-3 I2-S will be compared and analyzed. In addition, the a priori SRP models to augment the ECOM1 model will be derived based on analytical cuboid box model and empirically spectra analysis. We prefer the a priori model, as the additionally introduced higher-order harmonics in ECOM2 may increase the sensitivity to other modeling errors and result in a slightly degraded performance during certain mission phase. For ABW model, problems of observability likewise affect its use, as there are quite strong correlations between the estimation parameters.

Following short descriptions of the yaw attitude and body structure of BeiDou-3 I2-S satellite, the ECOM1 and ECOM2 model, as well as the ABW and cuboid models will be presented in Section 2. In Section 3, the comparison and analysis of performance of these models for BeiDou-3 I2-S will be presented. Based on these results, an empirical a priori model to augment the ECOM1 will be established by fitting the reconstructed SRP acceleration to the predetermined formulae after spectral density analysis with fast Fourier transformation (FFT) approach in Section 4. In addition, the parameters for the cuboid model will also be presented. Afterwards, the developed models will be validated in Section 5. Finally, this study is summarized and concluded in Section 6.

\section{Characteristics and SRP Models for BeiDou-3 I2-S Satellite}

The details of the optical and geometrical properties, as well as the attitude of satellite bus and solar panels (SP), are essential for the modeling of non-gravitational forces. In this section, these data as well as SRP models for BeiDou-3 I2-S satellite will be presented. 


\subsection{Attitude}

The estimated yaw attitudes of the BeiDou-3 I2-S satellite demonstrated that the continuous yaw attitude has been adopted instead of orbital normal mode used by BeiDou-2 IGSO and MEO satellites when BeiDou-3 I2-S is in eclipse season [3], and that the satellite experienced midnight- or noon-turn maneuvers when the sun elevation angle above the orbital plane ( $\beta$ angle) was in the range of $\left[-3^{\circ}\right.$, $\left.+3^{\circ}\right]$ and the orbital angle was in the range of approximately $\left[-6^{\circ}, 6^{\circ}\right]$ or $\left[174^{\circ}, 186^{\circ}\right]$. The midnightand noon-turn yaw maneuver model have been established by [12], but not been used in this study. For other period, the nominal yaw attitude is used to describe the orientation of BeiDou-3 I2-S; in this case, the satellite body-fixed frame $\left(\mathbf{e}_{S A T, X}, \mathbf{e}_{S A T, Y}, \mathbf{e}_{S A T, Z}\right)$ is defined by the three vectors, respectively:

$$
\mathbf{e}_{S A T, X}=\mathbf{e}_{S A T, Y} \times \mathbf{e}_{S A T, Z} \mathbf{e}_{S A T, Y}=\frac{\mathbf{e}_{\odot} \times \mathbf{r}}{\left\|\mathbf{e}_{\odot} \times \mathbf{r}\right\|} \mathbf{e}_{S A T, Z}=-\frac{\mathbf{r}}{\|\mathbf{r}\|},
$$

where $\mathbf{r}$ is the geocentric vector of the satellite and $\mathbf{e}_{\odot}$ is the unit vector pointing from the satellite to the Sun.

\subsection{Satellite Structure}

As described in [3], BeiDou-3 experimental satellites are based on two different satellite platforms developed by China Academy of Space Technology (CAST) and the China Academy of Science (CAS). The CAS platform weighs approximately $848 \mathrm{~kg}$, and has an elongated shape. On the other hand, the CAST IGSO platform has a cuboid shape and weighs $2800 \mathrm{~kg}$. BeiDou-3 I2-S is believed to be developed based on CAST's Dongfanghong-3A bus, which was also adopted by BeiDou-2 satellites. However, the structure of the satellite bus has changed to approximately $2 \times 2.5 \times 3.6 \mathrm{~m}$. As there are no available optical properties for BeiDou-3 I2-S, the coarse values from BeiDou-2 IGSO and MEO satellites have been used. Table 1 listed these initial values for satellite bus and SPs. As can been seen, the body of BeiDou-3 I2-S is also an elongated shape along Z axis, similar to Galileo and QZSS Michibiki.

Table 1. The coarse values of optical (absorption $\alpha$, reflectivity $\rho$, and diffusion $\delta$ ) and geometrical properties of satellite bus and SPs for BeiDou-3 I2-S satellite.

\begin{tabular}{ccccc}
\hline Panel & Area $\left[\mathrm{m}^{2}\right]$ & $\alpha$ & $\rho$ & $\delta$ \\
\hline$+\mathrm{X}$ & 7.20 & 0.350 & 0.650 & 0.0 \\
$-\mathrm{X}$ & 7.20 & 0.350 & 0.650 & 0.0 \\
$+\mathrm{Y}$ & 9.00 & 0.114 & 0.856 & 0.0 \\
$-\mathrm{Y}$ & 9.00 & 0.114 & 0.856 & 0.0 \\
$+\mathrm{Z}$ & 5.00 & 0.350 & 0.650 & 0.0 \\
$-\mathrm{Z}$ & 5.00 & 0.350 & 0.650 & 0.0 \\
SPs & 40.56 & 0.720 & 0.280 & 0.0 \\
\hline
\end{tabular}

\subsection{ECOM Model}

As an empirical SRP model, the ECOM model was originally proposed in the 1990s [4], and further develops the reduced and extended version $[5,10]$. The ECOM model decomposes the SRP acceleration into three orthogonal directions: the satellite-to-Sun direction $\left(\mathbf{e}_{D}\right)$, the nominal SP axis in YS mode $\left(\mathbf{e}_{Y}\right)$, and the orthogonal axis $\left(\mathbf{e}_{B}\right)$

$$
\begin{gathered}
\mathbf{e}_{D}=\mathbf{e}_{\odot} \\
\mathbf{e}_{Y}=\frac{\mathbf{e}_{\odot} \times \mathbf{r}}{\left\|\mathbf{e}_{\odot} \times \mathbf{r}\right\|} \\
\mathbf{e}_{B}=\mathbf{e}_{D} \times \mathbf{e}_{Y} . \\
a_{D}=D_{0}+D_{c} \cos \mu+D_{S} \sin \mu \\
a_{Y}=Y_{0}+Y_{c} \cos \mu+Y_{S} \sin \mu \\
a_{B}=B_{0}+B_{c} \cos \mu+B_{S} \sin \mu,
\end{gathered}
$$


Within ECOM model, the empirical SRP accelerations in the three directions at 1 astronomical unit (AU) are expressed as Equation (3) in a right-handed DYB frame for YS attitude in term of three constant accelerations $\left(D_{0}, Y_{0}\right.$, and $\left.B_{0}\right)$ and six one-cycle-per-revolution (1-cpr) accelerations (Dc, Ds, $Y_{c}, Y_{s}, B c$, and $\left.B s\right)$, where $\mu$ is the orbit angle; i.e., the argument of satellite with respect to the midnight point, i.e., the position where the satellite is far away from the Sun. Although this model can incorporate with an a priori model, good performance can also be obtained without such a priori model. Hence, the empirical reduced 5-parameter ECOM (called ECOM1 hereafter) is widely used to generate GNSS orbits. In this case, only three constant accelerations and two 1-cpr accelerations in $B$ direction are estimated.

In recent years, however, it became obvious that the ECOM1 could introduce large draconitic error in GNSS geodetic products [13,14]. In order to reduce the error in the time series of IGS (International GNSS Service) products, ECOM2 model has been proposed by additional introduction of high-order harmonics in Equation (3) [10], and expressed as

$$
\begin{aligned}
& a_{D}=D_{0}+\sum_{i=1}^{n_{D}}\left\{D_{2 i, c} \cos (2 i \Delta u)+D_{2 i, s} \sin (2 i \Delta u)\right\} \\
& a_{Y}=Y_{0} \\
& a_{B}=B_{0}+\sum_{i=1}^{n_{B}}\left\{B_{2 i-1, c} \cos ((2 i-1) \Delta u)+B_{2 i-1, s} \sin ((2 i-1) \Delta u)\right\}
\end{aligned}
$$

where the upper limit values, $n_{D}, n_{Y}$, and $n_{B}$ are defined by users, and $D_{2 i, c}, D_{2 i, s}, B_{2 i-1, c}$, and $B_{2 i-1, s}$ are the SRP coefficients to be estimated, and $\Delta u$ is the difference of the satellite's argument of the latitude and the Sun's argument of the latitude. As recommendation by [10], the 2-cpr and 4-cpr terms in $D$ and the 1-cpr terms in $B$ direction is better to account for.

\subsection{Box-Wing Model}

Based on the analytical physical theory, the SRP acceleration generated by a surface of area A depends on the relative alignment of the Sun direction and the surface normal $\mathbf{e}_{N}$ as well as the fraction of absorbed photons $(\alpha)$, of diffusely reflected photons $(\delta)$, and of specularly reflected photons $(\rho)[15,16]$. For $\cos \theta=\mathbf{e}_{\odot}^{T} \cdot \mathbf{e}_{N}>0$, that is, for an illuminated surface, the acceleration $\boldsymbol{a}$ at a distance of 1 AU can be accounted as

$$
a=-\frac{A}{M} \frac{S_{0}}{c} \cos \theta\left[(1-\rho) \mathbf{e}_{\odot}+2\left(\frac{\delta}{3}+\rho \cos \theta\right) \mathbf{e}_{N}\right],
$$

where $S_{0}=1367 \mathrm{~W} / \mathrm{m}^{2}$ is the solar flux at $1 \mathrm{AU}, c$ is the vacuum velocity of light, and $M$ is the total mass of the satellite. This equation can be used for SRP calculation on SPs. For a satellite bus panel, which is covered by multilayer insulation for thermal protection, the thermal re-radiation must be accounted for. Hence, the modified formulation for the acceleration [17] on the satellite bus surfaces is

$$
a=-\frac{A}{M} \frac{S_{0}}{c} \cos \theta\left[(\alpha+\delta)\left(\mathbf{e}_{\odot}+\frac{2}{3} \mathbf{e}_{N}\right)+2 \rho \cos \theta \cdot \mathbf{e}_{N}\right]
$$

By simply treating the satellite as a combination of box as two wings, the SRP acceleration acting on the satellite can be easily calculated by summing the SRP accelerations of each illustrated satellite bus panels and SPs.

As the changes in the a priori geometrical or optical properties of the satellite, or attitude deviations from the nominal, result in deficiencies of the above analytical model in modeling actual SRP perturbation, the adjustable box-wing model (ABW) has been proposed [17]. By fitting the optical coefficients and other parameters with real-tracking measurements, this model provides precise orbit solution with a clear physical understanding of SRP. For the satellites with nominal YS attitude, up to nine parameters are adjusted, including the absorption plus diffusion $(\alpha+\delta)$ as well as reflection $(\rho)$ for 
the illustrated surfaces $(+X,+Z$, and $-Z)$, scale parameter of SPs $\left(1+\rho+\frac{2}{3} \delta\right)$, SP rotation lag (denoted as SB hereafter), and $Y$ bias. From Equation (6), the partial derivatives of the acceleration w.r.t the optical properties of the satellite surfaces can be obtained as

$$
\begin{aligned}
& \frac{\partial a}{\partial(\alpha+\delta)}=-\frac{A}{M} \frac{S_{0}}{c} \cos \theta\left(\mathbf{e}_{\odot}+\frac{2}{3} \mathbf{e}_{N}\right), \\
& \frac{\partial a}{\partial \rho}=-\frac{A}{M} \frac{S_{0}}{c} 2 \cos ^{2} \theta \cdot \mathbf{e}_{N} .
\end{aligned}
$$

For the nominal YS attitude, the SPs are perpendicular to the Sun direction with $\cos \theta=1$ and $\mathbf{e}_{N}=\mathbf{e}_{\odot}$, the partial derivatives of the acceleration w.r.t scale parameter of SPs and SB are expressed as:

$$
\begin{gathered}
\frac{\partial a}{\partial\left(1+\rho+\frac{2}{3} \delta\right)}=-\frac{A}{M} \frac{S_{0}}{c} \mathbf{e}_{\odot}, \\
\frac{\partial a}{\partial S B}=-\frac{A}{M} \frac{S_{0}}{c} 2\left(\frac{\delta}{3}+\rho\right) \operatorname{sign}(\dot{\varepsilon}) \mathbf{e}_{B} .
\end{gathered}
$$

The correlation between parameters of ABW model is strong, so reasonable results are achieved only with a priori constraints being put on most of those parameters. To avoid the disadvantages of the ABW model, the box-wing model was reformulated [6]. The deduced model employs a specific parameterization that isolates distinct contributions of the satellite bus, in which the SRP accelerations depends only on the $\varepsilon$ angle. Without considering the SRP acting on SPs, the model can be expressed in $D$ and $B$ directions in the DYB frame as

$$
\begin{aligned}
a_{D}= & -a_{C}^{\alpha \delta}\left(|\cos \varepsilon|+\sin \varepsilon+\frac{2}{3}\right)-a_{S}^{\alpha \delta}\left(|\cos \varepsilon|-\sin \varepsilon-\frac{4}{3} \sin ^{2} \varepsilon+\frac{2}{3}\right) \\
& -a_{A}^{\alpha \delta}\left(\cos \varepsilon+\frac{2}{3}|\cos \varepsilon| \cos \varepsilon\right)-2 a_{C}^{\rho}\left(|\cos \varepsilon| \cos ^{2} \varepsilon+\sin ^{3} \varepsilon\right) \\
& -2 a_{C}^{\rho}\left(|\cos \varepsilon| \cos ^{2} \varepsilon-\sin ^{3} \varepsilon\right)-2 a_{A}^{\rho} \cos ^{3} \varepsilon \\
a_{B}= & -\frac{4}{3} a_{S}^{\alpha \delta}(\cos \varepsilon \sin \varepsilon)-\frac{2}{3} a_{A}^{\alpha \delta}(|\cos \varepsilon| \sin \varepsilon) \\
& -2 a_{C}^{\rho}((|\cos \varepsilon|-\sin \varepsilon) \cos \varepsilon \sin \varepsilon)-2 a_{S}^{\rho}((|\cos \varepsilon|+\sin \varepsilon) \cos \varepsilon \sin \varepsilon) \\
& -2 a_{A}^{\rho} \cos ^{2} \varepsilon \sin \varepsilon
\end{aligned}
$$

where $a_{C}^{\alpha \delta}, a_{S}^{\alpha \delta}$ and $a_{A}^{\alpha \delta}$ indicate the effect of a cubic $(C)$, stretched $(S)$, and asymmetric $(A)$ contributions of satellite body shape related to absorption plus diffuse reflection (superscript $\alpha \delta$ ), while model parameters $a_{C}^{\rho}, a_{S}^{\rho}$ and $a_{A}^{\rho}$ are related to the specular reflection $(\rho)$ part for the corresponding body shape contributions, and the above parameters can be obtained by,

$$
\begin{array}{ll}
a_{i}^{\alpha \delta}=\frac{A_{i}}{M} \frac{S_{0}}{c}\left(\alpha_{i}+\delta_{i}\right) & a_{i}^{\rho}=\frac{A_{i}}{M} \frac{S_{0}}{c} \rho_{i} \\
a_{z}^{\alpha \delta}=\frac{1}{2}\left(a_{+z}^{\alpha \delta}+a_{-z}^{\alpha \delta}\right) & a_{z}^{\rho}=\frac{1}{2}\left(a_{+z}^{\rho}+a_{-z}^{\rho}\right) \\
a_{C}^{\alpha \delta}=\frac{1}{2}\left(a_{z}^{\alpha \delta}+a_{+x}^{\alpha \delta}\right) & a_{C}^{\rho}=\frac{1}{2}\left(a_{z}^{\rho}+a_{+x}^{\rho}\right) \\
a_{S}^{\alpha \delta}=\frac{1}{2}\left(a_{z}^{\alpha \delta}-a_{+x}^{\alpha \delta}\right) & a_{S}^{\rho}=\frac{1}{2}\left(a_{z}^{\rho}-a_{+x}^{\rho}\right) \\
a_{A}^{\alpha \delta}=\frac{1}{2}\left(a_{+z}^{\alpha \delta}-a_{-z}^{\alpha \delta}\right) & a_{A}^{\rho}=\frac{1}{2}\left(a_{+z}^{\rho}-a_{-z}^{\rho}\right)
\end{array}
$$

where $a_{i}^{\alpha \delta}$ and $a_{i}^{\rho}(i=+Z,-Z,+X)$ are related to absorption plus diffuse reflection and reflection of the individual satellite body surface. These parameters are calculated from the a priori geometrical information list in Table 1. The Sun elongation angle $\varepsilon$ in Equation (10) can be expressed as

$$
\cos \varepsilon=\cos \beta \cos \mu
$$

\section{Performance of SRP Models for BeiDou-3 I2-S Satellite}

The POD strategy used has already been presented in [3]. The differences to be addressed here included the data period and tracking stations used. In general, the data collected by 25 stations from 11 November 2016 to 15 September 2017 have been processed. The increase in the number of available tracking stations is mainly contributed to the upgrades of the receivers from International 
GNSS Monitoring and Assessment system (iGMAS) network. Three solutions, named ECOM1, ECOM2, and ABW were determined according to the SRP models used. The SLR and orbit boundary discontinuities (OBD) have been used for evaluating the orbit quality. SLR residuals exceeding an absolute value of $60 \mathrm{~cm}$ were excluded. As $72 \mathrm{~h}$ POD arc used, the OBD are obtained as the three-dimensional (3D) orbit difference at midnight epoch between two 3-day arc shifted by $72 \mathrm{~h}$. In addition, the OBDs were treated as outliers and removed once the 3D values of the orbit differences were larger than $200 \mathrm{~cm}$.

Figure 1 shows the one-way SLR residuals in the function of the $\varepsilon$ angle for the three solutions. In expectation, similar as that shown in [3], the SLR residuals of ECOM1 solution show pronounced $\varepsilon$-angle-dependent variation with bias up to $-16.9 \mathrm{~cm}$. Once the ECOM2 has been used, the bias has been reduced to $-8.9 \mathrm{~cm}$ with slightly degraded standard derivations from 16.9 to $19.0 \mathrm{~cm}$. However, most importantly, the $\varepsilon$-angle-dependent error has been reduced. It is worth noticing that the $\varepsilon$ angle is actually a function of both $\beta$ and $\mu$, as expressed in Equation (12). We therefore decomposed the SLR residuals into the two arguments respectively as shown in Figure 2, and found that a marked v-shaped SLR variation with symmetry to the zero degree of $\mu$ angle can be identified for the ECOM1 solution, while it did not exist in ECOM2 solution, while the dependence with to $\beta$ angle is not clear as the $\mu$ angle. This might be attributed to smaller value of ratio of area-to-mass and less extended body shape used by BeiDou-3 I2-S satellite. For ABW solution, which shows the best SLR validation, the bias of SLR residuals was further reduced to $-2.2 \mathrm{~cm}$, and possibly generated by the unmodeled earth radiation pressure and antenna thrust. In addition, there are almost no significant $\varepsilon$-angle-dependent variations in SLR residuals. This further demonstrates that ABW has the ability to capture the SRP perturbation quite well.

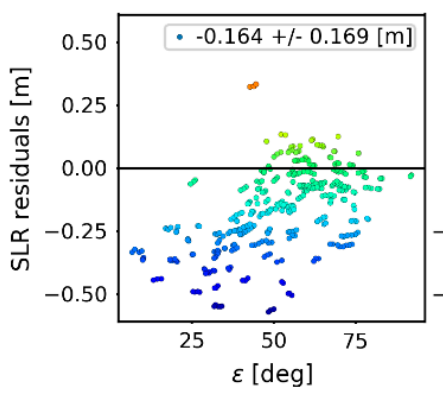

(a)

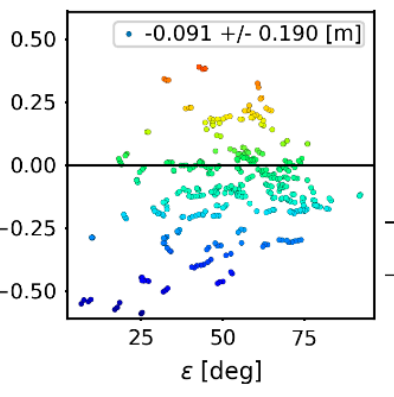

(b)

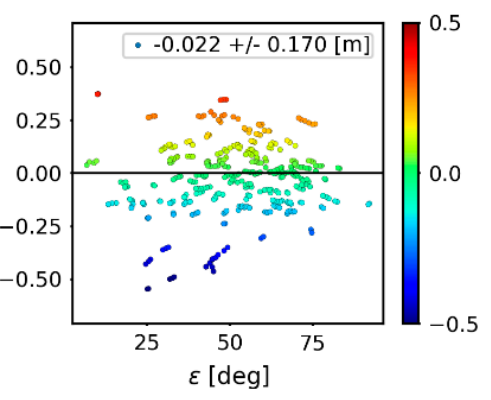

(c)

Figure 1. One-way satellite laser ranging (SLR) residuals of (a) Extended CODE Orbit Model (ECOM1), (b) extended ECOM model (ECOM2), and (c) adjustable box-wing model (ABW) orbit solutions of BeiDou-3 I2-S satellite with respect to the $\varepsilon$ angle.

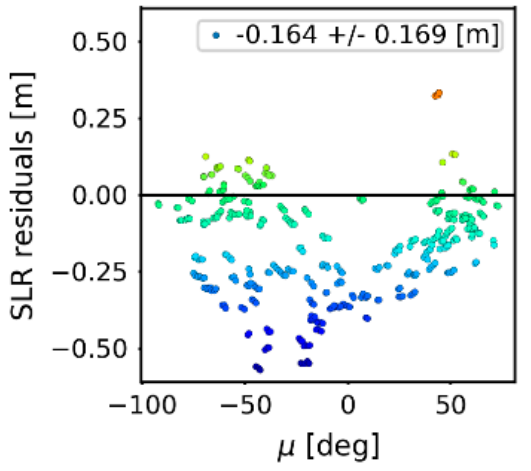

(a)

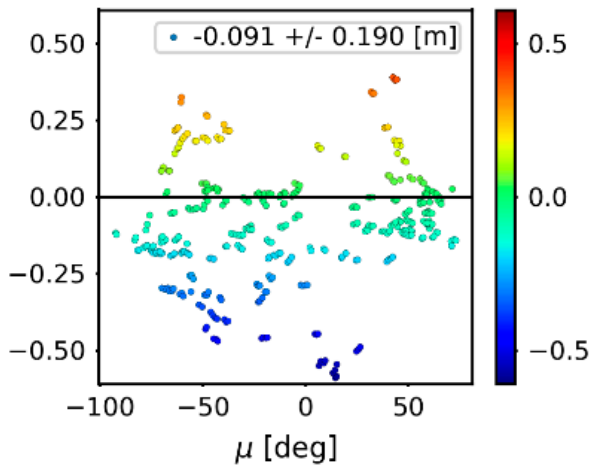

(b)

Figure 2. One-way SLR residuals of (a) ECOM1 and (b) ECOM2 orbit solutions of BeiDou-3 I2-S satellite with respect to the $\mu$ angle. 
Figure 3 shows the daily OBD values in along-track, cross-track, and radial direction for these three solutions, and Table 2 lists the mean values. It is worth noticing that some days were excluded as orbit maneuvers were detected, e.g., 25 August and 20 September 2017. In general, the three solutions show almost same performance, although ABW has slightly better performance in along-track and radial direction. The large OBDs in cross-track direction might be related to some onboard tests in March 2017. The radial OBDs show $\beta$ angle dependent variations, and became larger with an increase of $\beta$ angle. In addition, the slightly worse OBDs have been identified in eclipse periods, in particular for ECOM2 solution in radial direction and ABW solution in along-track direction. For ABW solution, it is possibly caused by deviations between the nominal yaw attitude and the real one, as we did not model the yaw maneuvers.

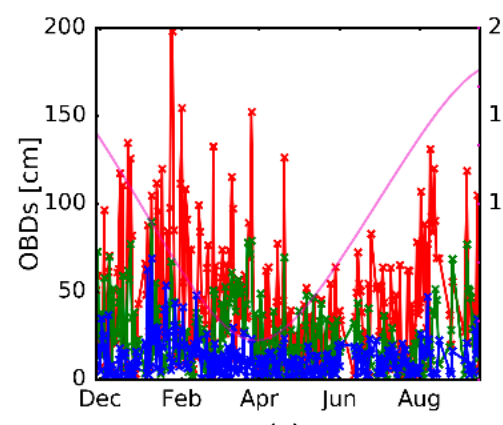

(a)

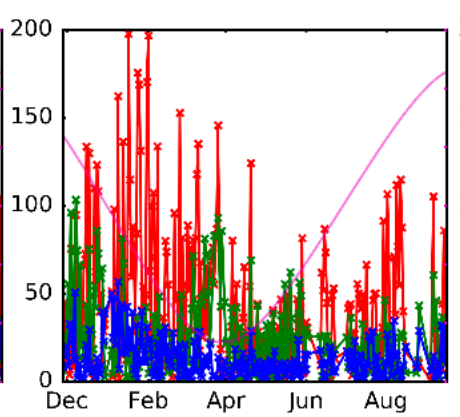

(b)

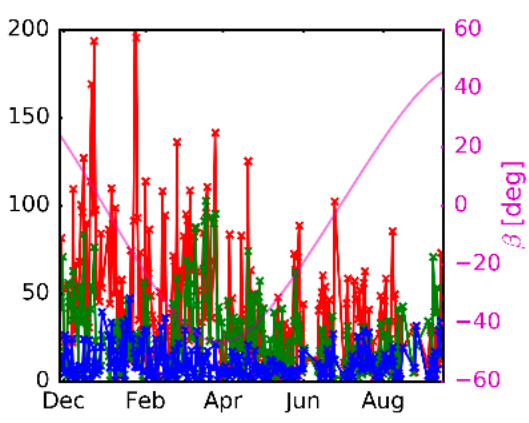

(c)

Figure 3. Time series of orbit boundary discontinuities (OBDs) for (a) ECOM1, (b) ECOM2 and (c) ABW solutions of BeiDou-3 I2-S. The red, green and blue curves represent OBDs in along-track, cross-track and radial direction, respectively.

Table 2. Mean value of OBDs in Along-track, Cross-track, radial and 3D for BeiDou-3 I2-S ECOM1, ECOM2 and ABW solutions. Values in brackets was for eclipse season (unit: $\mathrm{cm}$ ).

\begin{tabular}{ccccc}
\hline Solution & Along-Track & Cross-Track & Radial & 3D \\
\hline ECOM1 & $44.1(44.7)$ & $23.8(22.4)$ & $11.5(12.6)$ & $51.4(51.6)$ \\
ECOM2 & $42.1(42.6)$ & $25.2(26.1)$ & $12.4(18.6)$ & $50.6(53.4)$ \\
ABW & $41.8(54.1)$ & $26.2(20.5)$ & $10.6(11.0)$ & $50.5(58.9)$ \\
\hline
\end{tabular}

The above results demonstrated that better orbit consistency and accuracy could be achieved by use of ABW model. In addition, the $\varepsilon$-angle-dependent error can also be reduced by using ABW model, hence, it is possible to establish a better SRP model for the BeiDou-3 I2-S satellite based on ABW model, as we did for QZSS Michibiki mission [11].

\section{The a Priori SRP Models for BeiDou-3 I2-S Satellite}

With the above considerations, two a priori models will be developed in this section to augment the ECOM1.

\subsection{Analytical Cuboid Box Model}

The cuboid box model, as expressed in Equation (10), was employed in a specific parameterization, which separates the contribution of an ideal cube from that of a cuboid and the $\pm z$ asymmetry. By analyzing the correlation between the parameters and their contributions to the SRP perturbation, only two independent parameters have been selected to simplify the model as

$$
\begin{aligned}
& a_{D}=-a_{C}^{\alpha \delta}\left(|\cos \varepsilon|+\sin \varepsilon+\frac{2}{3}\right)-a_{S}^{\alpha \delta}\left(|\cos \varepsilon|-\sin \varepsilon-\frac{4}{3} \sin ^{2} \varepsilon+\frac{2}{3}\right) \\
& a_{B}=-\frac{4}{3} a_{S}^{\alpha \delta}(\cos \varepsilon \sin \varepsilon)
\end{aligned}
$$


As the first one cannot be separated from the contribution of SPs. Hence, the value is calculated from the optical and geometrical parameter listed in Table 1. The second one was adjusted from a least-squares adjustment based on the Equation (13). Table 3 lists the estimated values for these two parameters.

Table 3. Values for cuboid box model parameters (unit: $\mathrm{nm} / \mathrm{s}^{2}$ ).

\begin{tabular}{cc}
\hline Para. & Value \\
\hline$a_{C}^{\alpha \delta}$ & 11.9 \\
$a_{S}^{\alpha \delta}$ & 2.1 \\
\hline
\end{tabular}

\subsection{Empirical SRP Model}

In addition, the purely empirical parameter fitting approach was used to develop an a priori SRP model for BeiDou-3 I2-S satellite based on the reconstructed SRP acceleration from ABW solution.

The SRP accelerations for BeiDou-3 I2-S satellite were reconstructed by orbit integration with the determined orbital elements and SRP parameters from the ABW solution, and were decomposed in DYB frame. Figure 4 illustrates the amplitude spectra of the reconstructed SRP acceleration for $\beta$ angle about $10^{\circ}, 30^{\circ}$, and $45^{\circ}$ in DYB frame. The strength of spectra line for 0 -cpr signal in $D$ reaches to about $-100 \mathrm{~nm} / \mathrm{s}^{2}$, where it is about $1 \mathrm{~nm} / \mathrm{s}^{2}$ in $B$ direction. For other periodic signals, the sizeable spectral lines exist for even orders in $D$. However, for $B$ axis the sizeable spectral lines exist for odd orders in B. Similar results were obtained for GPS and GLONASS [10]. A significant 1-cpr signal in $B$ with amplitudes of about $2 \mathrm{~nm} / \mathrm{s}^{2}$ can be identified. The accelerations along $Y$ are not presented here as the order of that is as small as $0.1 \mathrm{~nm} / \mathrm{s}^{2}$.

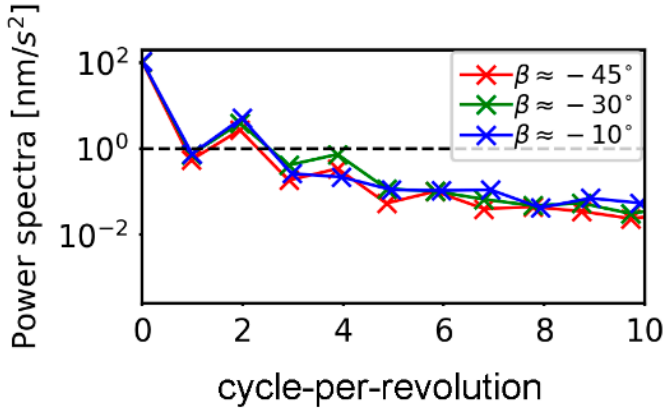

(a)

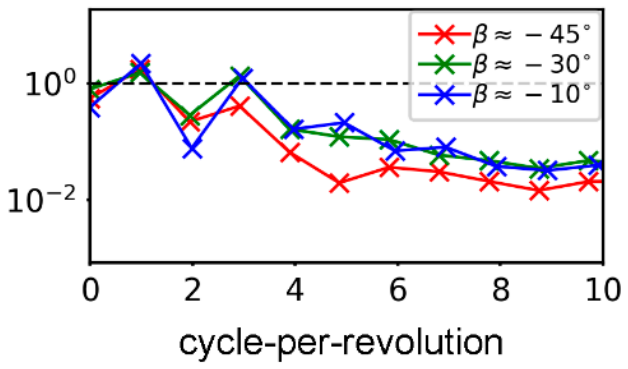

(b)

Figure 4. Power spectra of the reconstructed SRP accelerations of ABW solutions in (a) $D$ and (b) $B$ directions, respectively. (The dashed line in the above figures refers to $1 \mathrm{~nm} / \mathrm{s}^{2}$ ).

Based on the above spectrum analysis, the periodic signals with amplitudes of over $0.6 \mathrm{~nm} / \mathrm{s}^{2}$ are selected to present the SRP perturbation acting on BeiDou-3 I2-S satellite. These are 2-cpr as well as 4-cpr signal in $D$, and 1-cpr, as well as 3-cpr signal in $B$. Hence, the a priori model can be expressed as,

$$
\begin{aligned}
& a_{D, a p}=D_{0, a p}+D_{2 c} \cos (2 \varepsilon)+D_{4 c} \cos (4 \varepsilon) \\
& a_{B, a p}=B_{0, a p}+B_{1 c} \cos (\varepsilon)+B_{3 c} \cos (3 \varepsilon)
\end{aligned}
$$

where $D_{0, a p}, D_{2 c}, D_{4 c}, B_{0, a p}, B_{1 c}$, and $B_{3 c}$ are the model coefficients. Here, and after, we called this empirical reconstructed a priori SRP model as FFT model.

Afterwards, the coefficients in Equation (14) were estimated by fitting with the daily reconstructed accelerations in each axes. Clear $\beta$-angle-dependent variation for $D_{0, a p}$ and $D_{2 c}$ could be observed, as shown in Figure 5. The estimations of $D_{0, a p}$ and $D_{2 c}$ show parabola variations against $\beta$ angle with $0^{\circ}$ vertices. These indicate that the model needs to be improved further with accounting for such 
variations. To model them, we fitted these parameters with the 2-order polynomial against the $\beta$ angle for $D_{0, a p}$ and $D_{2 c}$. The derived coefficients for the model are listed in Table 4.

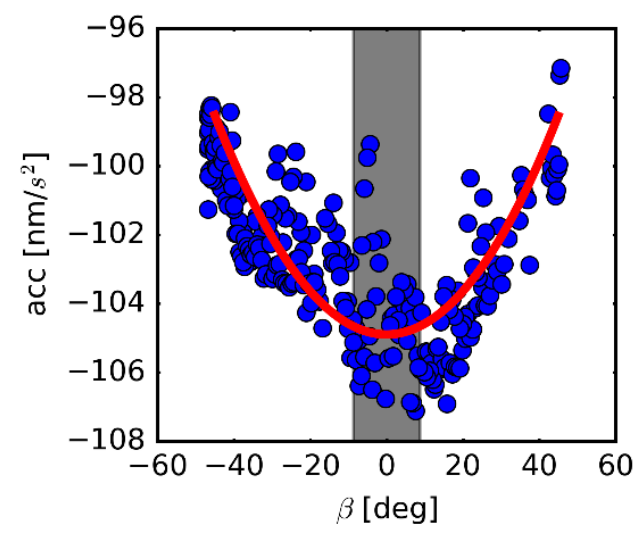

(a)

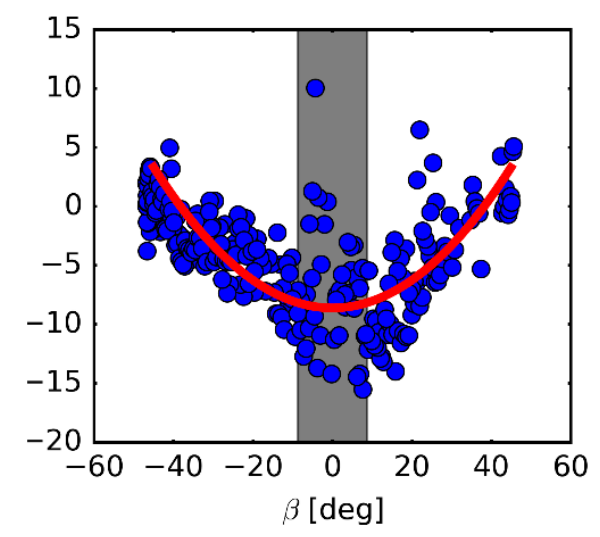

(b)

Figure 5. Variations of the model values for (a) and (b) $D_{2 c}$. Shadowed area for eclipse season.

Table 4. Values for the empirical a priori SRP model expressed in Equation (14) (unit: $\mathrm{nm} / \mathrm{s}^{2}, \beta$ in degrees).

\begin{tabular}{cccccc}
\hline Parameters & $\boldsymbol{D}_{0, a p}$ & $\boldsymbol{D}_{2 c}$ & $\boldsymbol{D}_{4 c}$ & $\boldsymbol{B}_{1 c}$ & $\boldsymbol{B}_{3 c}$ \\
\hline value & $3.05 \mathrm{e}-3 \times \beta^{2}-105$ & $5.9 \mathrm{e}-3 \times \beta^{2}-8.62$ & 0.96 & 1.6 & 0.6 \\
\hline
\end{tabular}

\section{Validation}

The same data set were used to validate the performance of the above proposed two a priori models. Two solutions were determined by using cuboid model or empirical FFT model. The SLR and OBD are also used to validate the solutions' quality.

Figure 6 illustrates the SLR residuals of these two solutions against the $\varepsilon$ angle. In general, once the proposed models used as the priori models of ECOM1, the remarkable improvements can be obtained. For all of the two solutions with the a priori model, the $\varepsilon$-angle-dependent error was almost vanished, while the $-17.0 \mathrm{~cm}$ bias was reduced to $-6.1 \mathrm{~cm}$ and $-4.0 \mathrm{~cm}$, respectively. Between the two solutions, almost same performance was obtained, though the SLR residuals of empirical FFT model became slightly scatter and has a little larger standard derivation.

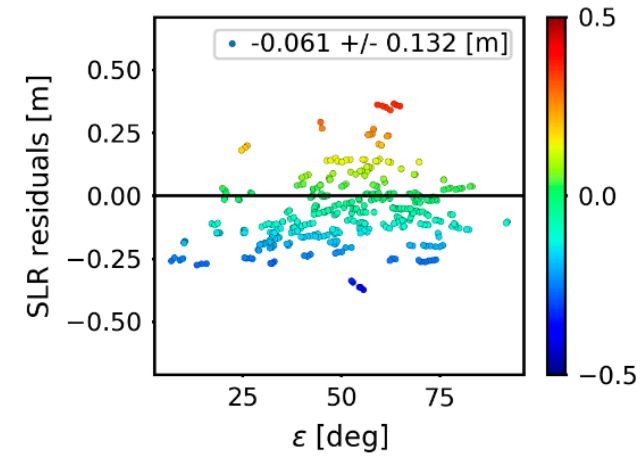

(a)

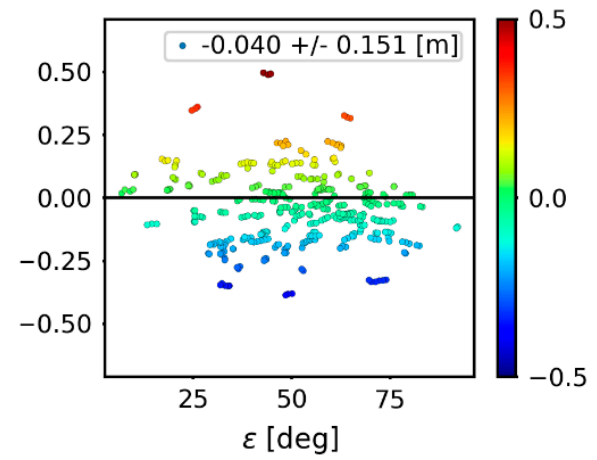

(b)

Figure 6. One-way SLR residuals of solutions using the a priori (a) cuboid model and (b) fast Fourier transformation (FFT) model for BeiDou-3 I2-S with respect to $\varepsilon$ angle. 
Time series of daily OBDs for the two solutions are illustrated in Figure 7, and Table 5 lists the mean values in Along-track, Cross-track, and radial directions. For both solutions, almost identical OBDs are achieved, and show similar performance as that of ECOM1 solution. The radial OBDs show $\beta$ angle dependent variations, and the worse OBDs have been identified in eclipse periods. For Galileo satellites with a priori model for ECOM1 [6], the OBDs also have similar performance as that of solution determined with ECOM1 only.

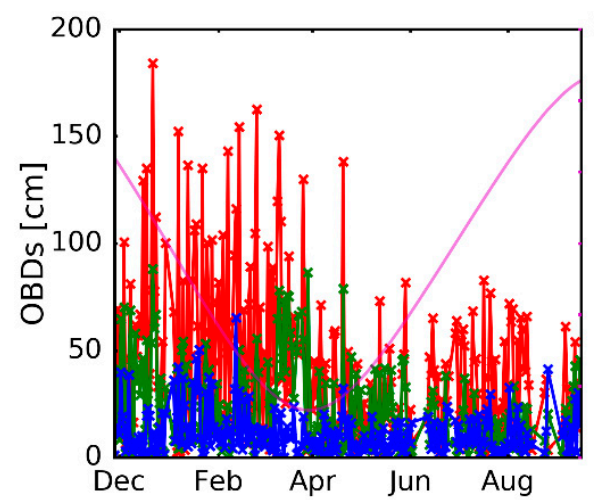

(a)

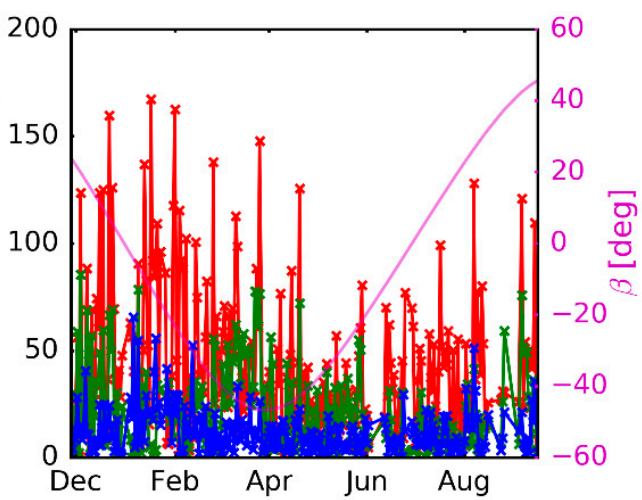

(b)

Figure 7. Time series of OBDs for solutions using the a priori (a) cuboid model and (b) FFT model for BeiDou-3 I2-S. The red, green and blue curves represent OBDs in along-track, cross-track and radial direction, respectively.

Table 5. Mean value of OBDs in Along-track, Cross-track, radial, and three-dimensional (3D) for BeiDou-3 I2-S solutions with cuboid and priori model to argument ECOM1. Values in brackets was for eclipse season (unit: $\mathrm{cm}$ ).

\begin{tabular}{ccccc}
\hline Solution & Along-Track & Cross-Track & Radial & 3D \\
\hline Cuboid + ECOM1 & $42.4(43.5)$ & $22.5(22.1)$ & $11.0(12.1)$ & $49.3(50.3)$ \\
FFT + ECOM1 & $42.6(43.5)$ & $21.5(17.0)$ & $11.8(12.4)$ & $49.1(48.4)$ \\
\hline
\end{tabular}

\section{Conclusions}

Similar to Galileo satellites, deficiency in ECOM1 orbit solution for BeiDou-3 I2-S satellites was identified, in which a marked $\varepsilon$-angle-dependent radial error exists. The standalone ECOM1 fail to properly describe the acceleration acting on an extended body shape with a large area-to-mass ratio. Through the comparisons of the widely used SRP models i.e., ECOM1, ECOM2, and ABW, we found that all of them showed similar orbit accuracy of approximately $50 \mathrm{~cm}$ revealed by OBDs, but SLR validation results of ECOM1 confirmed larger $\varepsilon$-angle-dependent error, as well as a bias of nearly $-17 \mathrm{~cm}$. Although the $\varepsilon$-angle-dependent errors in SLR residuals can be reduced by ECOM2, there was still a large bias. Fortunately, the ABW model can be used to overcome the issues.

We established an analytical a priori cuboid model from coarse information of BeiDou-3 I2-S satellite. In addition, the empirical FFT model was obtained with accuracy of around $1 \mathrm{~nm} / \mathrm{s}^{2}$. By augmenting the ECOM1 with the a priori models, radial orbit accuracy was improved by approximately $35 \%$ w.r.t the ECOM1 solution. More importantly, the $\varepsilon$-angle-dependent error of SLR residuals was almost eliminated.

Though the success of the applications of those priori models, further efforts should be performed for the orbit dynamics in eclipse seasons. Yaw attitude is another point to be carefully considered to model SRP. In addition, relevant modeling of SRP by ray-tracking method [18] is in need for the detailed structure and the additional effects of shadowing and secondary intersections. 
Acknowledgments: The IGS MGEX (Multi-GNSS Experiment), iGMAS (International GNSS Monitoring and Assessment System), and ILRS (International Laser Ranging Service) are greatly acknowledged for providing the multi-GNSS and SLR tracking data. The research is partially supported by the National Natural Science Foundation of China (Grant No. 41504009, 41574030).

Author Contributions: Chen Wang, Jing Guo and Qile Zhao provided the initial idea for this study. Jing Guo designed the experiments. Wang performed the experiments. Chen Wang and Jing Guo analyzed the data; Qile Zhao contributed to the analysis tools. Jingnan Liu and Qile Zhao gave valuable advice on the paper writing.

Conflicts of Interest: The authors declare no conflict of interest.

\section{References}

1. Yang, D.; Yang, J.; Li, G.; Zhou, Y.; Tang, C. Globalization highlight: Orbit determination using BeiDou inter-satellite ranging measurements. GPS Solut. 2017, 21, 1395-1404. [CrossRef]

2. Zhang, X.; Wu, M.; Liu, W.; Li, X.; Yu, S.; Lu, C.; Wickert, J. Initial assessment of the COMPASS/BeiDou-3: New-generation navigation signals. J. Geodesy 2017, 91, 1225-1240. [CrossRef]

3. Zhao, Q.; Wang, C.; Guo, J.; Liu, J. Precise orbit and clock determination for BeiDou-3 experimental satellites with yaw attitude analysis. GPS Solut. 2018, 22, 4. [CrossRef]

4. Beutler, G.; Brockmann, E.; Gurtner, W.; Hugentobler, U.; Mervart, L.; Rothacher, M. Extended orbit modeling techniques at the CODE processing center of the International GPS Service for Geodynamics (IGS): Theory and initial results. Manuscr. Geod. 1994, 19, 367-386.

5. Springer, T.A.; Beutler, G.; Rothacher, M. A new solar radiation pressure model for GPS satellites. GPS Solut. 1999, 3, 50-62. [CrossRef]

6. Montenbruck, O.; Steigenberger, P.; Hugentobler, U. Enhanced solar radiation pressure modeling for galileo satellites. J. Geodesy 2015, 89, 283-297. [CrossRef]

7. Steigenberger, P.; Montenbruck, O. Galileo status: Orbits, clocks, and positioning. GPS Solut. 2016, 21, 319-331. [CrossRef]

8. Montenbruck, O.; Steigenberger, P.; Darugna, F. Semi-Analytical Solar Radiation Pressure Modeling for QZS-1 Orbit-Normal and Yaw-Steering Attitude. Adv. Space Res. 2017, 59, 2088-2100. [CrossRef]

9. Prange, L.; Orliac, E.; Dach, R.; Arnold, D.; Beutler, G.; Schaer, S.; Jäggi, A. CODE's five-system orbit and clock solution-The challenges of multi-GNSS data analysis. J. Geodesy 2016, 91, 345-360. [CrossRef]

10. Arnold, D.; Meindl, M.; Beutler, G.; Dach, R.; Schaer, S.; Lutz, S.; Prange, L.; Sośnica, K.; Mervart, L.; Jäggi, A. CODE's new solar radiation pressure model for GNSS orbit determination. GPS Solut. 2015, 89, 775-791. [CrossRef]

11. Zhao, Q.; Chen, G.; Guo, J.; Liu, J.; Liu, X. An a priori solar radiation pressure model for the QZSS Michibiki satellite. J. Geodesy 2017, 1-13. [CrossRef]

12. Wang, C.; Guo, J.; Zhao, Q. Precise orbit and clock determination for BeiDou-3 experimental satellites with yaw attitude analysis: Latest developments (in Chinese). In Proceedings of the 5th International GNSS Monitoring and Assessment System Technical Symposium, Sanya, China, 16 November 2017.

13. Meindl, M.; Beutler, G.; Thaller, D.; Jäggi, A.; Dach, R. Geocenter coordinates estimated from GNSS data as viewed by perturbation theory. Adv. Space Res. 2013, 51, 1047-1064. [CrossRef]

14. Rodríguez-Solano, C.J.; Hugentobler, U.; Steigenberger, P.; Blossfeld, M.; Fritsche, M. Reducing the draconitic errors in GNSS geodetic products. J. Geodesy 2014, 88, 559-574. [CrossRef]

15. Milani, A.; Nobili, A.M.; Farinella, P. Non-Gravitational Perturbartions and Satellite Geodesy; Adam Hilger: Bristol, UK, 1987.

16. Fliegel, H.; Gallini, T.; Swift, E. Global Positioning System Radiation Force Model for Geodetic Applications. J. Geophys. Res. 1992, 97, 559-568. [CrossRef]

17. Rodriguez-Solano, C.J.; Hugentobler, U.; Steigenberger, P. Adjustable box-wing model for solar radiation pressure impacting GPS satellites. Adv. Space Res. 2012, 49, 1113-1128. [CrossRef]

18. Ziebart, M. Generalized analytical solar radiation pressure modeling algorithm for spacecraft of complex shape. J. Spacecr. Rocket. 2004, 41, 840-848. [CrossRef]

(C) 2018 by the authors. Licensee MDPI, Basel, Switzerland. This article is an open access article distributed under the terms and conditions of the Creative Commons Attribution (CC BY) license (http:// creativecommons.org/licenses/by/4.0/). 